\title{
SMALL MOTIONS OF IDEAL STRATIFIED LIQUID WITH A FREE SURFACE TOTALLY COVERED BY A CRUMBLED ICE
}

\author{
N.D. KOPACHEVSKY, D.O. TSVETKOV
}

\begin{abstract}
Let a rigid immovable vessel be partially filled with an ideal incompressible stratified fluid. We assume that in an equilibrium state the density of a fluid is a function of the vertical variable $x_{3}$, i.e., $\rho_{0}=\rho_{0}\left(x_{3}\right)$. In this case the gravity field with constant acceleration $\vec{g}=-g \vec{e}_{3}$ acts on the fluid, here $g>0$ and $\vec{e}_{3}$ is unit vector of the vertical axis $O x_{3}$, which is directed opposite to $\vec{g}$. Let $\Omega$ be the domain filled with a fluid in equilibrium state, $S$ be rigid wall of the vessel adherent to the fluid, $\Gamma$ be a free surface completely covered with a crumbled ice. As the crumbled ice we mean that on the free surface, weighty particles of some substance float, and these particles do not interact or the interaction is negligible as the free surface oscillates. We should note that in foreign publications, such fluids are frequently called liquids with inertial free surfaces. The problem is studied on the base of an approach connected with application of so-called operator matrices theory. To this end, we introduce Hilbert spaces and some their subspaces, also auxiliary boundary value problems. The initial boundary value problem is reduced to the Cauchy problem for the differential second-order equation in Hilbert space. After a detailed study of the properties of the operator coefficients corresponding to the resulting system of equations, we prove a theorem on the strong solvability of the Cauchy problem obtained on a finite time interval. On this base, we find sufficient conditions for the existence of a strong (with respect to time variable) solution to the initial-boundary value problem describing the evolution of the hydrosystem.
\end{abstract}

Keywords: stratification effect in ideal fluids, initial boundary value problem, differential equation in Hilbert space, Cauchy problem, strong solution.

Mathematics Subject Classifcation: 35D35

\section{INTRODUCTION}

An important part of a general problem on dynamics of a body with a cavity containing a liquid is a problem on motion of a liquid in a stationary vessel. Let us briefly mention the problems related directly with the subject of the present work.

The problems on oscillations of a stratified liquid filling a bounded domain in a space have applications in the seiche theory, the theory of petroleum oscillations in tankers, in studying oscillations of cryogenic liquids in closed tanks. Not providing a detailed bibliography, we just mention monographs [1]-[6] and works [7], [8], where various aspects of oscillations of such system were studied. It is known that the presence of a vertical stratification of a liquid with respect to the density gives rise quite to interesting physical phenomena in such hydrosystems; these phenomena are related with the action of buoyancy forces. For instance, in the oceans

N.D. Kopachevsiy, D.O. Tsvetkov, Small motions of ideal Stratified liquid With a fReE SuRFACE TOTALLY COVERED BY A CRUMBLED ICE.

(C) Kopachevsiy N.D.,TsvetKov D.O. 2018.

Submitted June 29, 2017. 
these forces generate internal inertial waves of large amplitude, which can cause catastrophes. In tankers filled by the petroleum, there can arise oscillations leading to an unstable motion of a ship.

An ice cover is an important part of a hydrological regime of freezing seas and oceans. The presence of calved ice on the surface of seas and oceans influence essentially their behaviour. Study of such problems is one of the important part of oceanology, its practical efficiency is undoubted. A problem close to the problem on dynamics of liquids in domain covered by a crumbled ice called the problem on floatation was studied in works [9], [10, and also in thesis [11.

\section{Mathematical Formulation of PROBlem}

We consider an ideal stratified liquid whose density $\rho_{0}$ varies along the vertical axis $O x_{3}$ in the quiescent state, $\rho_{0}=\rho_{0}\left(x_{3}\right)$. The liquid fills partially a stationary vessel and in the quiescent state, it fills a domain $\Omega$ bounded by a hard wall $S$ and a free surface $\Gamma$ totally covered by a crumbled ice. We assume that the origin $O$ of the Cartesian coordinate system $O x_{1} x_{2} x_{3}$ is taken on a free equilibrium surface $\Gamma$ which is flat and is located perpendicular to the acceleration of the gravity $\vec{g}=-g \vec{e}_{3}$, where $\vec{e}_{3}$ is the unit vector of the axis $O x_{3}$. We also assume that the hard wall $S \subset \partial \Omega$ is a Lipschitz surface and $\partial S=\partial \Gamma$ is a Lipschitz curve.

We consider the main case of a stable stratification of the liquid with respect to the density:

$$
\begin{aligned}
& 0<N_{\text {min }}^{2} \leqslant N^{2}\left(x_{3}\right) \leqslant N_{\text {max }}^{2}=N_{0}^{2}<\infty, \\
& N^{2}\left(x_{3}\right)=-\frac{g \rho_{0}^{\prime}\left(x_{3}\right)}{\rho_{0}\left(x_{3}\right)}, \quad \rho_{0}(0)>0 .
\end{aligned}
$$

The function $N\left(x_{3}\right)$ is called Brunt-Väisälä frequency or buoyancy frequency. Physically, $N\left(x_{3}\right)$ is equal to the frequency of oscillations made by a particle of a liquid at $x_{3}=$ const in the stratified liquid once this particle is moved from this level.

We consider small linear motions of the stratified liquid close to the equilibrium. By $\vec{u}$ we denote the field of velocities of the liquid in $\Omega$. Then the motion of the hydrosystem is described by the following equation (see [12]):

$$
\frac{\partial \vec{u}}{\partial t}+(\vec{u} \cdot \nabla) \vec{u}=-\widehat{\rho}^{-1} \cdot \nabla P+\vec{F} \quad(\text { in } \Omega),
$$

where $\widehat{\rho}(t, x), P(t, x)$ and $\vec{F}(t, x)$ are total fields of the density, pressure in the liquid and the field of external forces, respectively.

We should also add a continuity equation or a mass conservation law. It expresses an obvious fact that the mass of the liquid in the volume enveloping the same particles conserves (see [13]):

$$
\frac{\partial \widehat{\rho}}{\partial t}+\operatorname{div}(\widehat{\rho} \vec{u})=0 \quad(\text { in } \Omega) .
$$

Let us determine the statical pressure in the liquid. If the system is in the equilibrium state and only the gravity force acts on it, $\vec{g}=-g \vec{e}_{3}$, then $\vec{F}=\vec{g}$ and by (2) with $\vec{u}=\overrightarrow{0}$ we obtain

$$
\nabla P_{0}=-\rho_{0}\left(x_{3}\right) g \overrightarrow{e_{3}} .
$$

This is why the static pressure $P_{0}(x)$ in the liquid is equal to

$$
P_{0}(x)=P_{0}\left(x_{3}\right)=p_{a}-g \int_{0}^{x_{3}} \rho_{0}(\zeta) d \zeta
$$

where $p_{a}$ is the constant external pressure. 
Considering the problem on small motions of the system, we represent the fields $P(t, x)$, $\widehat{\rho}(t, x)$ and $\vec{F}(t, x)$ as

$$
P(t, x)=P_{0}(x)+p(t, x), \quad \widehat{\rho}(t, x)=\rho_{0}\left(x_{3}\right)+\rho(t, x), \quad \vec{F}(t, x)=\vec{f}(t, x)-g \vec{e}_{3},
$$

where $p(t, x)$ is the deviation of the pressure field from the equilibrium field $P_{0}\left(x_{3}\right), \rho(t, x)$ is the deviation of the density field from the initial one $\rho_{0}\left(x_{3}\right)$, and $\vec{f}(t, x)$ is a small field of external mass forces.

We substitute representations (5) into equations (2), (3):

$$
\begin{gathered}
\frac{\partial \vec{u}}{\partial t}+(\vec{u} \cdot \nabla) \vec{u}=-\frac{1}{\rho_{0}\left(x_{3}\right)+\rho(t, x)} \nabla\left(P_{0}\left(x_{3}\right)+p(t, x)\right)+\vec{f}(t, x)-g \vec{e}_{3} \quad(\text { in } \Omega), \\
\frac{\partial}{\partial t}\left(\rho_{0}\left(x_{3}\right)+\rho(t, x)\right)+\operatorname{div}\left(\left(\rho_{0}\left(x_{3}\right)+\rho(t, x)\right) \vec{u}\right) \\
=\frac{\partial \rho}{\partial t}+\nabla \rho_{0} \cdot \vec{u}+\nabla \rho \cdot \vec{u}+\left(\rho_{0}+\rho\right) \operatorname{div} \vec{u}=0 \quad(\text { in } \Omega) .
\end{gathered}
$$

Taking into consideration that the density does not vary with the time, that is,

$$
\frac{d \widehat{\rho}}{d t}=\frac{\partial \rho}{\partial t}+\nabla \rho_{0} \cdot \vec{u}+\nabla \rho \cdot \vec{u}=0
$$

linearizing (6), (7), we obtain

$$
\begin{aligned}
& \frac{\partial \vec{u}}{\partial t}=\rho_{0}^{-1}\left(x_{3}\right)\left(-\nabla p-g \rho \vec{e}_{3}\right)+\vec{f} \quad(\text { in } \Omega), \\
& \frac{\partial \rho}{\partial t}+\nabla \rho_{0} \cdot \vec{u}=0, \quad \operatorname{div} \vec{u}=0 \quad(\operatorname{in} \Omega) .
\end{aligned}
$$

Since we consider the motion of the liquid in a hard vessel, on its hard wall $S$, the ideal liquid should obey the non-leaking condition:

$$
\vec{u} \cdot \vec{n}=: u_{n}=0 \quad(\text { on } S),
$$

where $\vec{n}$ is the unit outward normal to the domain $\Omega$.

We proceed to identifying a kinematic condition on the equilibrium surface $\Gamma$. We seek the deviation of a moving surface $\Gamma(t)$ as

$$
\Gamma(t): \quad x_{3}=\zeta=\zeta(t, \hat{x}), \quad \hat{x}:=\left(x_{1}, x_{2}\right) \in \Gamma .
$$

In this case the linearized kinematic conditions for the particles of the liquid located at $\Gamma(t)$ reads as

$$
\frac{\partial \zeta}{\partial t}=u_{n}:=\vec{u} \cdot \vec{n} \quad(\text { on } \Gamma)
$$

Writing the second Newton law for the particles of the crumbled ice and linearizing it, we obtain the second dynamical condition (see [9]):

$$
p=g \rho_{0}(0) \zeta+\rho_{2} \frac{\partial^{2} \zeta}{\partial t^{2}} \quad(\text { on } \Gamma)
$$

where $\rho_{2}$ is the surface density of the crumbled ice. By crumbled ice we mean floating on the free surface weighty particles of some matter, which do not interact while floating or their interaction is negligible. At that, the particle are located on the surface all the time during the small motions of this hydrodynamical system. 
Thus, a linear formulation of the problem on oscillations of the considered hydrosystem is as follows:

$$
\begin{aligned}
& \frac{\partial \vec{u}}{\partial t}=\rho_{0}^{-1}\left(x_{3}\right)\left(-\nabla p-g \rho \vec{e}_{3}\right)+\vec{f}(t, x) \quad(\text { in } \Omega), \\
& \operatorname{div} \vec{u}=0, \quad \frac{\partial \rho}{\partial t}+\nabla \rho_{0} \cdot \vec{u}=0 \quad(\operatorname{in} \Omega), \\
& \vec{u} \cdot \vec{n}=: u_{n}=0 \quad(\text { on } S), \quad u_{n}=\frac{\partial \zeta}{\partial t} \quad(\text { on } \Gamma), \quad \int_{\Gamma} \zeta d \Gamma=0, \\
& p=g \rho_{0}(0) \zeta+\rho_{2} \frac{\partial^{2} \zeta}{\partial t^{2}} \quad(\text { on } \Gamma), \\
& \vec{u}(0, x)=\vec{u}^{0}(x), \quad \rho(0, x)=\rho^{0}(x) \quad(x \in \Omega), \quad \zeta(0, \hat{x})=\zeta_{0}(\hat{x}) \quad(\hat{x} \in \Gamma) .
\end{aligned}
$$

The latter three identities are initial conditions completing the problem and $\int_{\Gamma} \zeta d \Gamma=0$ is the volume conservation condition.

We note that a classical solution to problem (13) obeys the law of total energy balance:

$$
\begin{aligned}
\frac{1}{2} \cdot & \frac{d}{d t}\left[\left(\int_{\Omega} \rho_{0}\left(x_{3}\right)|\vec{u}|^{2} d \Omega+\rho_{2} \int_{\Gamma}\left|\frac{\partial \zeta}{\partial t}\right|^{2} d \Gamma\right)\right. \\
& \left.+\left(g^{2} \int_{\Omega}\left[\rho_{0}\left(x_{3}\right) N^{2}\left(x_{3}\right)\right]^{-1}|\rho|^{2} d \Omega+g \rho_{0}(0) \int_{\Gamma}|\zeta|^{2} d \Gamma\right)\right]=\int_{\Omega} \rho_{0}\left(x_{3}\right) \vec{f} \cdot \vec{u} d \Omega .
\end{aligned}
$$

The left hand side is the sum of the derivative in $t$ of the total kinetic energy of the system and its potential energy. The total potential energy (the expression in second brackets) is equal to the sum of the potential energy due to the presence of buoyancy forces and to the potential energy due to the oscillations of free surface. The right hand side is the power of external forces.

\section{EXClusion OF DENSITY FIELD. USING OF FIELD OF LIQUID SMALL SHIFTS}

In initial boundary value problem (13) we can exclude one unknown function, the density field $\rho(t, x)$ if instead of the velocity field $\vec{u}(t, x)$ we introduce the field of liquid small shifts $\vec{v}(t, x)$ related with $\vec{u}(t, x)$ by the identities:

$$
\frac{\partial \vec{v}}{\partial t}=\vec{u}, \quad \operatorname{div} \vec{v}=0 \quad(\text { in } \Omega)
$$

Then instead of (8) and (9) we arrive at the constraint:

$$
\begin{aligned}
& \rho(t, x)=-\nabla \rho_{0} \cdot \vec{v}(t, x)+f_{0}(x)=-\rho_{0}^{\prime}\left(x_{3}\right) v_{3}(t, x)+f_{0}(x), \\
& f_{0}(x):=\rho(0, x)+\rho_{0}^{\prime}\left(x_{3}\right) v_{3}(0, x), \quad v_{3}:=\vec{v} \cdot \vec{e}_{3}
\end{aligned}
$$

and to the equations for $\vec{v}(t, x)$ and $p(t, x)$ :

$$
\begin{aligned}
& \frac{\partial^{2} \vec{v}}{\partial t^{2}}=-\rho_{0}^{-1}\left(x_{3}\right) \nabla p-N^{2}\left(x_{3}\right) v_{3} \vec{e}_{3}+\psi_{0}(x), \quad \operatorname{div} \vec{v}=0 \quad(\text { in } \Omega), \\
& \psi_{0}(x)=\vec{f}(t, x)-g f_{0}(x) \vec{e}_{3} / \rho_{0}\left(x_{3}\right) .
\end{aligned}
$$


In view of the said above we rewrite original problem $(13)$ as

$$
\begin{aligned}
& \frac{\partial^{2} \vec{v}}{\partial t^{2}}=-\rho_{0}^{-1}\left(x_{3}\right) \nabla p-N^{2}\left(x_{3}\right) v_{3} \vec{e}_{3}+\psi_{0}(x), \quad \operatorname{div} \vec{v}=0 \quad(\text { in } \Omega), \\
& \vec{v} \cdot \vec{n}=: v_{n}=0 \quad(\text { on } S), \quad p=g \rho_{0}(0) v_{3}+\rho_{2} \frac{\partial^{2} v_{3}}{\partial t^{2}} \quad(\text { on } \Gamma), \\
& \frac{\partial \vec{v}}{\partial t}(0, x)=\vec{u}(0, x)=\vec{u}^{0}(x), \quad \vec{v}(0, x)=\vec{v}^{0}(x), \\
& v_{3}(0, \hat{x})=\zeta(0, \hat{x})=\zeta^{0}(\hat{x}) \quad(\hat{x} \in \Gamma) .
\end{aligned}
$$

Initial boundary value problem (18) involves only two unknown functions: the vector field $\vec{v}(t, x)$ and the scalar field of pressures $p(t, x)$. Given the solution $\vec{v}(t, x)$ to problem (18), the solutions $\vec{u}(t, x)$ and $\rho(t, x)$ to problem $(13)$ can be found by formulae (15) and (16).

\section{Projection of MOtion EQUATIONS ON ORTHOGONAL SPACES}

In what follows we reduce initial boundary value problem (18) to a differential equation in a Hilbert space. In order to do this, we employ projecting of the first equation in (18) on orthogonal subspaces, see [15]. To the function $\rho_{0}$, we associate the Hilbert space $\vec{L}_{2}\left(\Omega, \rho_{0}\right)$ of vector functions with the scalar product

$$
(\vec{u}, \vec{v})=\int_{\Omega} \rho_{0}\left(x_{3}\right) \vec{u}(x) \overline{\vec{v}(x)} d \Omega .
$$

As it follows from (1), the function $\rho=\rho_{0}\left(x_{3}\right)$ satisfy the inequalities

$$
0<m \leqslant \rho_{0} \leqslant M<\infty
$$

ensuring the equivalence of the norm defined by $(19)$ and the usual scalar product in $\vec{L}_{2}(\Omega)$.

By $\vec{J}_{0}\left(\Omega, \rho_{0}\right)$ we denote a subspace in $\vec{L}_{2}\left(\Omega, \rho_{0}\right)$ obtained as the closure of the set of smooth functions

$$
\left\{\vec{v} \in \vec{C}^{1}(\Omega): \operatorname{div} \vec{v}=0(\text { in } \Omega), v_{n}=0(\text { on } \partial \Omega)\right\}
$$

in the norm of $\vec{L}_{2}\left(\Omega, \rho_{0}\right)$.

As other subspaces, we choose

$$
\begin{aligned}
& \left.\vec{G}_{h, S}\left(\Omega, \rho_{0}\right)=\left\{\vec{v} \in \vec{L}_{2}\left(\Omega, \rho_{0}\right): \vec{v}=\rho_{0}^{-1} \nabla p, v_{n}=0 \text { (on } S\right), \nabla \cdot \vec{v}=0(\text { in } \Omega), \int_{\Gamma} p d \Gamma=0\right\}, \\
& \vec{G}_{0, \Gamma}\left(\Omega, \rho_{0}\right)=\left\{\vec{w} \in \vec{L}_{2}\left(\Omega, \rho_{0}\right): \vec{w}=\rho_{0}^{-1} \nabla \varphi, \quad \varphi=0(\text { on } \Gamma)\right\}
\end{aligned}
$$

We observe that here $v_{n}=\rho_{0}^{-1} \partial p / \partial n$ is a functional in the space $\widetilde{H}_{S}^{-1 / 2}=\left(H_{S}^{1 / 2}\right)^{*}$ consisting of the functions, which can be continued by zero in the class $H^{-1 / 2}(\partial \Omega)$ on the entire $\partial \Omega$, at that, $H^{1 / 2}(\partial \Omega) \subset L_{2}(\partial \Omega) \subset H^{-1 / 2}(\partial \Omega)$ (see, [14, Ch. 3]).

Lemma 1. The following orthogonal expansion holds:

$$
\vec{L}_{2}\left(\Omega, \rho_{0}\right)=\vec{J}_{0}\left(\Omega, \rho_{0}\right) \oplus \vec{G}_{h, S}\left(\Omega, \rho_{0}\right) \oplus \vec{G}_{0, \Gamma}\left(\Omega, \rho_{0}\right) .
$$

The proof of the lemma reproduces the proof of a similar statement in [15] for the space $\vec{L}_{2}(\Omega)$ with $\rho_{0}\left(x_{3}\right)=$ const in $19 p$.

We shall regard $\vec{u}(t, x)$ and $\rho_{0}^{-1} \nabla p(t, x)$ as functions of the variable $t$ with values in $\vec{L}_{2}\left(\Omega, \rho_{0}\right)$, then by the equation and boundary conditions in (18) and orthogonal expansion (20) we have

$$
\vec{v}(t, x) \in \vec{J}_{0}\left(\Omega, \rho_{0}\right) \oplus \vec{G}_{h, S}\left(\Omega, \rho_{0}\right)=: \vec{J}_{0, S}\left(\Omega, \rho_{0}\right),
$$




$$
\rho_{0}^{-1} \nabla p(t, x) \in \vec{G}_{0, \Gamma}\left(\Omega, \rho_{0}\right) \oplus \vec{G}_{h, S}\left(\Omega, \rho_{0}\right)=: \vec{G}\left(\Omega, \rho_{0}\right) .
$$

This is why for each $t$ we seek them as

$$
\begin{aligned}
& \vec{v}(t, x)=\vec{w}(t, x)+\rho_{0}^{-1} \nabla \Phi(t, x), \vec{w}(t, x) \in \vec{J}_{0}\left(\Omega, \rho_{0}\right), \rho_{0}^{-1} \nabla \Phi(t, x) \in \vec{G}_{h, S}\left(\Omega, \rho_{0}\right), \\
& \rho_{0}^{-1} \nabla p(t, x)=\rho_{0}^{-1} \nabla p_{1}(t, x)+\rho_{0}^{-1} \nabla p_{2}(t, x), \\
& \rho_{0}^{-1} \nabla p_{1}(x, t) \in \vec{G}_{h, S}\left(\Omega, \rho_{0}\right), \quad \rho_{0}^{-1} \nabla p_{2}(t, x) \in \vec{G}_{0, \Gamma}\left(\Omega, \rho_{0}\right) .
\end{aligned}
$$

We denote by $P_{0}, P_{h, S}$ and $P_{0, \Gamma}$ the orthogonal projectors on the subspaces $\vec{J}_{0}\left(\Omega, \rho_{0}\right)$, $\vec{G}_{h, S}\left(\Omega, \rho_{0}\right), \vec{G}_{0, \Gamma}\left(\Omega, \rho_{0}\right)$, respectively. Then, substituting (21) into the first equation in (18) and applying orthogonal projectors, we obtain

$$
\begin{aligned}
& \frac{\partial^{2} \vec{w}}{\partial t^{2}}+P_{0}\left[N^{2}\left(x_{3}\right)\left(\rho_{0}^{-1} \frac{\partial \Phi}{\partial x_{3}}+w_{3}\right) \vec{e}_{3}\right]=P_{0} \psi_{0} \\
& \frac{\partial^{2}}{\partial t^{2}}\left(\rho_{0}^{-1} \nabla \Phi\right)+\rho_{0}^{-1} \nabla p_{1}+P_{h, S}\left[N^{2}\left(x_{3}\right)\left(\rho_{0}^{-1} \frac{\partial \Phi}{\partial x_{3}}+w_{3}\right) \vec{e}_{3}\right]=P_{h, S} \psi_{0}, \\
& \rho_{0}^{-1} \nabla p_{2}+P_{0, \Gamma}\left[N^{2}\left(x_{3}\right)\left(\rho_{0}^{-1} \frac{\partial \Phi}{\partial x_{3}}+w_{3}\right) \vec{e}_{3}\right]=P_{0, \Gamma} \psi_{0} .
\end{aligned}
$$

It follows from equation (24) that a component of the pressure field related to the term $\rho_{0}^{-1} \nabla p_{2}$ is determined only the field of vertical shift $v_{3}$ and by initial conditions. Therefore, it is sufficient to consider only first two relations and boundary condition with the replacing $p \rightarrow p_{1}$ since $p=p_{1}+p_{2}, p_{2}=0$ (on $\left.\Gamma\right)$.

In order to proceed from (22), (23) to a system of equations for two unknown functions, we introduce new elements:

$$
P_{h, S}\left[N^{2}\left(x_{3}\right) w_{3} \vec{e}_{3}\right]:=\rho_{0}^{-1} \nabla \Psi, \quad P_{h, S}\left[N^{2}\left(x_{3}\right) \rho_{0}^{-1} \frac{\partial \Phi}{\partial x_{3}} \vec{e}_{3}\right]:=\rho_{0}^{-1} \nabla \eta .
$$

Then (23) gives Cauchy-Lagrange integral:

$$
\frac{\partial^{2} \Phi}{\partial t^{2}}+p_{1}+\Psi+\eta-F=c(t) \quad(\text { in } \Omega),
$$

where $c(t)$ is an arbitrary function of the time, $P_{h, S} \psi_{0}=\rho_{0}^{-1} \nabla F$.

We consider (26) on $\Gamma$ and in view of (22)- 24), we can employ the identity

$$
\begin{aligned}
p_{1} & =g \rho_{0}(0) v_{3}+\rho_{2} \frac{\partial^{2} v_{3}}{\partial t^{2}}=g \rho_{0}(0)\left(\rho_{0}^{-1} \frac{\partial \Phi}{\partial x_{3}}+w_{3}\right)+\rho_{2} \frac{\partial^{2}}{\partial t^{2}}\left(\rho_{0}^{-1} \frac{\partial \Phi}{\partial x_{3}}+w_{3}\right) \\
& \left.=g \rho_{0}(0)\left(\rho_{0}^{-1} \frac{\partial \Phi}{\partial x_{3}}\right)+\rho_{2} \frac{\partial^{2}}{\partial t^{2}}\left(\rho_{0}^{-1} \frac{\partial \Phi}{\partial x_{3}}\right)=g \frac{\partial \Phi}{\partial x_{3}}+\rho_{2} \rho_{0}^{-1}(0) \frac{\partial^{2}}{\partial t^{2}}\left(\frac{\partial \Phi}{\partial x_{3}}\right) \quad \text { (on } \Gamma\right)
\end{aligned}
$$

to obtain

$$
\frac{\partial^{2} \Phi}{\partial t^{2}}+g \frac{\partial \Phi}{\partial x_{3}}+\rho_{2} \rho_{0}^{-1}(0) \frac{\partial^{2}}{\partial t^{2}}\left(\frac{\partial \Phi}{\partial x_{3}}\right)+\Psi+\eta=F+c(t) \quad(\text { on } \Gamma) .
$$

This identity and (22) give two equations for determining two unknown functions $\vec{w}(t, x)$ and $\Phi(t, x)$. At that, constraints 25) are taken into consideration as well as constraints following 
(22)-(24). Thus, we rewrite initial boundary value problem $(18)$ as

$$
\begin{aligned}
& \frac{\partial^{2} \vec{w}}{\partial t^{2}}+P_{0}\left[N^{2}\left(x_{3}\right)\left(\rho_{0}^{-1} \frac{\partial \Phi}{\partial x_{3}}+w_{3}\right) \vec{e}_{3}\right]=P_{0} \psi_{0} \quad(\text { in } \Omega), \\
& \operatorname{div} \vec{w}=0 \quad(\operatorname{in} \Omega), \quad \vec{w} \cdot \vec{n}=0 \quad(\text { on } \partial \Omega), \\
& \frac{\partial^{2} \Phi}{\partial t^{2}}+g \frac{\partial \Phi}{\partial x_{3}}+\rho_{2} \rho_{0}^{-1} \frac{\partial^{2}}{\partial t^{2}}\left(\frac{\partial \Phi}{\partial x_{3}}\right)+\Psi+\eta=F+c(t) \quad(\text { on } \Gamma), \\
& \nabla \cdot\left(\rho_{0}^{-1}(x) \nabla \Phi\right)=0 \quad(\operatorname{in} \Omega), \quad \rho_{0}^{-1}(x) \nabla \Phi \cdot \vec{n}=0 \quad(\text { on } S), \\
& \int_{\Gamma} \Phi d \Gamma=0, \quad \int_{\Gamma} \frac{\partial \Phi}{\partial x_{3}} d \Gamma=0, \\
& \frac{\partial}{\partial t} w(0, x)=P_{0} \vec{u}^{0}, \quad \frac{\partial}{\partial t}\left(\rho_{0}^{-1} \frac{\partial \Phi}{\partial x_{3}}(0, \hat{x})\right)_{\Gamma}=\left[\left(P_{h, S} \vec{u}^{0}(x)\right) \cdot \vec{n}\right]_{\Gamma}, \\
& \vec{w}(0, x)=P_{0} \vec{v}^{0}, \quad\left(\rho_{0}^{-1} \frac{\partial \Phi}{\partial x_{3}}(0, \hat{x})\right)_{\Gamma}=\zeta^{0}(\hat{x}) \quad(\hat{x} \in \Gamma) .
\end{aligned}
$$

We note that the deviation $\left.v_{3}\right|_{\Gamma}=\left(\rho_{0}^{-1} \frac{\partial \Phi}{\partial x_{3}}+w_{3}\right)_{\Gamma}$ of the particles of a moving surface should obey the condition of volume conserving under the oscillations:

$$
\int_{\Gamma} v_{3} d \Gamma=\int_{\Gamma}\left(\rho_{0}^{-1} \frac{\partial \Phi}{\partial x_{3}}+w_{3}\right) d \Gamma=0 \Longrightarrow \int_{\Gamma} \frac{\partial \Phi}{\partial x_{3}} d \Gamma=0
$$

since $\left.w_{3}\right|_{\Gamma}=0,\left.\rho_{0}^{-1}\right|_{\Gamma}=$ const.

\section{REDUCTION TO SYSTEM OF DIFFERENTIAL OPERATOR EQUATIONS}

Condition (29) is necessary for solvability of the following problem

\section{Auxiliary problem I (Neumann problem).}

$$
\begin{aligned}
& \nabla \cdot\left(\rho_{0}^{-1}(x) \nabla \Phi\right)=0 \quad(\text { in } \Omega), \quad \rho_{0}^{-1}(x) \nabla \Phi \cdot \vec{n}=0 \quad(\text { on } S), \\
& \rho_{0}^{-1}(0) \frac{\partial \Phi}{\partial x_{3}}=\psi \quad(\text { on } \Gamma), \quad \int_{\Gamma} \psi d \Gamma=0 .
\end{aligned}
$$

In the space $H_{0}=L_{2, \Gamma}:=L_{2}(\Gamma) \ominus\left\{1_{\Gamma}\right\}$ we introduce its framing as $H_{+} \subset H_{0} \subset H_{-}$, where $H_{+}=H^{1 / 2}(\Gamma) \cap H_{0}=: H_{\Gamma}^{1 / 2}, H_{-}=\left(H_{+}\right)^{*}=: \widetilde{H}_{\Gamma}^{-1 / 2}$. Here by $\widetilde{H}_{\Gamma}^{-1 / 2}$ we denote a space dual for $H_{\Gamma}^{1 / 2}$ with a central space $L_{2, \Gamma}$. In particular, $\widetilde{H}_{\Gamma}^{-1 / 2}$ consists of the elements in $H^{-1 / 2}(\Gamma)$, which can be continued by zero in the class $H^{-1 / 2}(\partial \Omega)$ (see [14, Ch. 3]).

We observe that in view of (14), the scalar product in $L_{2}(\Gamma)$ is of the form

$$
(\eta, \zeta)_{0}:=\rho_{0}(0) \int_{\Gamma} \eta(\hat{x}) \overline{\zeta(\hat{x})} d \Gamma .
$$

Lemma 2. For each $\psi \in H_{-}=\widetilde{H}_{\Gamma}^{-1 / 2}$, problem 30 in a domain $\Omega$ with a Lipschitz (piecewise smooth) boundary $\partial \Omega$ has the unique generalized solution $\Phi \in H_{h, S}^{1}\left(\Omega, \rho_{0}\right)$ and

$$
\|\Phi\|_{H_{h, S}^{1}\left(\Omega, \rho_{0}\right)} \leqslant c_{1}\|\psi\|_{\widetilde{H}_{\Gamma}^{-1 / 2}} .
$$

Here $H_{h, S}^{1}\left(\Omega, \rho_{0}\right)$ is the subspace of the space $H_{\Gamma}^{1}\left(\Omega, \rho_{0}\right)$ with the norm

$$
\|\Phi\|_{H_{\Gamma}^{1}\left(\Omega, \rho_{0}\right)}^{2}:=\int_{\Omega} \rho_{0}^{-1}|\nabla \Phi|^{2} d \Omega, \int_{\Gamma} \Phi d \Gamma=0 ;
$$


this subspace is formed by quasi-harmonic functions obeying Neumann condition on $S$.

A generalized solution $\Phi(x)$ to problem (30) satisfies the identity

$$
(\Phi, \Psi)_{H_{h, S}^{1}\left(\Omega, \rho_{0}\right)}=\left(\psi,\left.\Psi\right|_{\Gamma}\right)_{H_{0}} \quad \text { for all } \Psi \in H_{\Gamma}^{1}\left(\Omega, \rho_{0}\right) .
$$

The proof of this lemma is provided, for instance, in [15].

Auxiliary problem II (Neumann-Zaremba problem).

$$
\begin{aligned}
& \nabla \cdot\left(\rho_{0}^{-1}(x) \nabla \Phi\right)=0 \quad(\text { in } \Omega), \quad \rho_{0}^{-1}(x) \nabla \Phi \cdot \vec{n}=0 \quad(\text { on } S), \\
& \rho_{0}^{-1}(0) \Phi=\varphi \quad(\text { on } \Gamma), \quad \int_{\Gamma} \varphi d \Gamma=0 .
\end{aligned}
$$

Lemma 3. For each $\varphi \in H_{+}=H_{\Gamma}^{1 / 2}$, problem (34) has the unique generalized solution $\Phi \in H_{h, S}^{1}\left(\Omega, \rho_{0}\right)$ and

$$
\|\Phi\|_{H_{h, S}^{1}\left(\Omega, \rho_{0}\right)} \leqslant c_{2}\|\varphi\|_{H_{\Gamma}^{1 / 2}} .
$$

The proof of this lemma is provided, for instance, in [15].

According Gagliardo theorem (see [16]), the trace of the function $\Phi(x) \in H_{h, S}^{1}\left(\Omega, \rho_{0}\right)$ on the boundary $\partial \Omega$ belongs to $H^{1 / 2}(\partial \Omega)$ and this is why the norm in $H_{\Gamma}^{1 / 2}=H_{+}$can be chosen among equivalent forms so that

$$
\|\Phi\|_{H_{h, S}^{1}(\Omega)}=\|\varphi\|_{H_{\Gamma}^{1 / 2}}, \quad \text { for all } \forall \varphi \in H_{\Gamma}^{1 / 2},
$$

where $\Phi(x)$ is the solution to problem (34).

We suppose that $\psi=\left(\rho_{0}^{-1} \frac{\partial \Phi}{\partial x_{3}}\right)_{\Gamma}$ is a given function in the space $\widetilde{H}_{\Gamma}^{-1 / 2}$. Considering the Neumann problem with this function $\psi$, by Lemma 2 we obtain that

$$
\Phi(x)=T \psi \in H_{h, S}^{1}\left(\Omega, \rho_{0}\right),
$$

where $T: \widetilde{H}_{\Gamma}^{-1 / 2} \rightarrow H_{h, S}^{1}\left(\Omega, \rho_{0}\right)$ is a bounded linear operator, whose norm does not exceeds the constant $c_{1}>0$ in $(32)$.

We introduce the trace operator $\gamma_{\Gamma}$ : for each $\Phi(x) \in H_{h, S}^{1}\left(\Omega, \rho_{0}\right)$ we let

$$
\gamma_{\Gamma} \Phi:=\left.\Phi\right|_{\Gamma} .
$$

We note that the operator $\gamma_{\Gamma}$ is bounded as that from $H^{1}\left(\Omega, \rho_{0}\right)$ (and hence from the subspace $\left.H_{h, S}^{1}\left(\Omega, \rho_{0}\right)\right)$ into $H_{+}=H_{\Gamma}^{1 / 2}$ (see, for instance, [16]). This yields that

$$
\gamma_{\Gamma} \Phi=\left.\Phi\right|_{\Gamma}=\gamma_{\Gamma} T \psi=\gamma_{\Gamma} T\left(\rho_{0}^{-1} \frac{\partial \Phi}{\partial x_{3}}\right)_{\Gamma}=: C\left(\rho_{0}^{-1} \frac{\partial \Phi}{\partial x_{3}}\right)_{\Gamma}
$$

where the operator $C=\gamma_{\Gamma} T: \widetilde{H}_{\Gamma}^{-1 / 2}=H_{-} \rightarrow H_{\Gamma}^{1 / 2}=H_{+}$is linear and bounded.

Lemma 4. The restriction of the operator $C$ on $H_{0} \subset H_{-}$is a linear compact self-adjoint positive operator acting in the space $H_{0}$.

Proof. If $\psi \in H_{0}$, then $C \psi=\gamma_{\Gamma} T \psi \in H_{+}$. Since the space $H_{+}=H_{\Gamma}^{1 / 2}$ is compactly embedded into the space $H_{0}$, and $C$ is a bounded operator from $H_{0}$ into $H_{+}$, the operator $C: H_{0} \rightarrow H_{0}$ is compact.

To prove the symmetricity (self-adjointness) and positivity of the operator $C$ we assume that $\Psi \in H_{h, S}^{1}\left(\Omega, \rho_{0}\right) \subset H_{\Gamma}^{1}(\Omega)$ in identity 33 and $\Psi(x)$ is also a solution to auxiliary problem (30) for a given function $v \in H_{0}$. Then

$$
\Phi=T \psi, \quad \psi=\left(\rho_{0}^{-1} \frac{\partial \Phi}{\partial x_{3}}\right)_{\Gamma}, \quad \gamma_{\Gamma} \Phi=C \psi,
$$




$$
\Psi=T v, \quad v=\left(\rho_{0}^{-1} \frac{\partial \Psi}{\partial x_{3}}\right)_{\Gamma}, \quad \gamma_{\Gamma} \Psi=\left.\Psi\right|_{\Gamma}=C v,
$$

By these formulae and (33) we obtain

$$
(\psi, C v)_{H_{0}}=\int_{\Omega} \rho_{0}^{-1} \nabla \Phi \cdot \overline{\nabla \Psi} d \Omega=(C \psi, v)_{H_{0}}, \quad \text { for all } \quad w, v \in H_{0},
$$

which implies that $C=C^{*}$. Letting her $v=\psi, \Psi=\Phi$, we get

$$
(C \psi, \psi)_{H_{0}}=\int_{\Omega} \rho_{0}^{-1}|\nabla \Phi|^{2} d \Omega=\|\Phi\|_{H_{\Gamma}^{1}\left(\Omega, \rho_{0}\right)}^{2} \geqslant 0 .
$$

If $(C \psi, \psi)_{H_{0}}=0$, then $\Phi \equiv \Phi_{0}=$ const. Hence, by the normalization of the function $\Phi$

$$
\int_{\Gamma} \Phi_{0} d \Gamma=0
$$

we obtain that $\Phi \equiv 0$, and hence $\psi=\left(\rho_{0}^{-1} \frac{\partial \Phi}{\partial x_{3}}\right)_{\Gamma}=0$. Thus, the operator $C$ is positive in $H_{0}$.

Remark. The proven lemma implies also the following statement: the operator $C: H_{0} \rightarrow H_{0}$ has an inverse operator $C^{-1}$, which is an unbounded self-adjoint positive definite operator acting in $H_{0}$ with the domain $\mathcal{D}\left(C^{-1}\right)=\mathcal{R}(C)$ dense in $H_{0}$.

Remark. It follows from (38), (40) that

$$
\left(C^{-1} \varphi, \varphi\right)_{H_{0}}=\|\Phi\|_{H_{h, S}^{1}\left(\Omega, \rho_{0}\right)}^{2}, \quad \gamma_{\Gamma} \Phi=\varphi \in \mathcal{D}\left(C^{-1}\right),
$$

where $\Phi$ is the solution to problem (34). This implies that choosing the norm in $H_{\Gamma}^{1 / 2}=H_{+}$by (36), the operator $C^{-1 / 2}$ acts isometrically from $\mathcal{D}\left(C^{-1 / 2}\right)=H_{+}$into $H_{0}=H$ :

$$
\left\|C^{-1 / 2} \varphi\right\|_{H_{0}}^{2}=\|\varphi\|_{H_{\Gamma}^{1 / 2}}^{2}, \quad \forall \varphi \in H_{+} .
$$

Thus, the operator $C^{-1 / 2}$ maps $\mathcal{D}\left(C^{-1 / 2}\right)=H_{+}$into $H_{0}$, and the operator $C^{1 / 2}$ maps $H_{0}$ into $\mathcal{D}\left(C^{-1 / 2}\right)=H_{+}$isometrically. As it follows from the general of framed Hilbert spaces, the extension of the operator $C^{-1 / 2}$ (which will be denoted by the same symbol) with $\mathcal{D}\left(C^{-1 / 2}\right)=H_{+}$ on $H_{0}$ is an isometric operator mapping $H_{0}$ onto $H_{-}=\widetilde{H}_{\Gamma}^{-1 / 2}$. Respectively, the operator $C^{1 / 2}$, having been extended to $H_{-}$, maps isometrically $H_{-}$onto $H_{0}$.

According above constructions (see (37)), we rewrite system of equations (22) and (27) together projecting in addition (27) on $H_{0}$ :

$$
\begin{aligned}
& \frac{\partial^{2} \vec{w}}{\partial t^{2}}+P_{0}\left[N^{2}\left(x_{3}\right)\left(\rho_{0}^{-1} \frac{\partial \Phi}{\partial x_{3}}\right) \vec{e}_{3}\right]+P_{0}\left[N^{2}\left(x_{3}\right) w_{3} \vec{e}_{3}\right]=P_{0} \psi_{0} \\
& \frac{\partial^{2}}{\partial t^{2}}\left[C+\rho_{2} I\right]\left(\rho_{0}^{-1} \frac{\partial \Phi}{\partial x_{3}}\right)_{\Gamma}+g \rho_{0}\left(\rho_{0}^{-1} \frac{\partial \Phi}{\partial x_{3}}\right)_{\Gamma}+P_{H_{0}}(\Psi+\eta)=P_{H_{0}} F
\end{aligned}
$$

Taking into consideration (30), in (43) we make the change:

$$
\psi=\left(\rho_{0}^{-1} \frac{\partial \Phi}{\partial x_{3}}\right)_{\Gamma}=C^{-1 / 2} y, \quad y \in H_{0} .
$$


We suppose that $P_{H_{0}} F \in \mathcal{D}\left(C^{-1 / 2}\right)$ and we apply the operator $C^{-1 / 2}$ to the second equation in (43). This gives:

$$
\begin{aligned}
& \frac{\partial^{2} \vec{w}}{\partial t^{2}}+P_{0}\left[N^{2}\left(x_{3}\right)\left(\left(U C^{-1 / 2} y\right) \vec{e}_{3}\right) \vec{e}_{3}\right]+P_{0}\left[N^{2}\left(x_{3}\right) w_{3} \vec{e}_{3}\right]=P_{0} \psi_{0}, \\
& \frac{\partial^{2}}{\partial t^{2}}\left[I+\rho_{2} C^{-1}\right] y+g \rho_{0} C^{-1} y+C^{-1 / 2} P_{H_{0}}(\Psi+\eta)=C^{-1 / 2} P_{H_{0}} F .
\end{aligned}
$$

Here by $U: \widetilde{H}_{\Gamma}^{-1 / 2} \rightarrow \vec{G}_{h, S}\left(\Omega, \rho_{0}\right)$ we denote the operator, which by means of the solution to problem 30 maps an element $\psi \in \widetilde{H}_{\Gamma}^{-1 / 2}$ into the function $\rho_{0}^{-1} \nabla \Phi \in \vec{G}_{h, S}\left(\Omega, \rho_{0}\right)$.

We introduce the following notations:

$$
\begin{aligned}
& B_{11} \vec{w}:=P_{0}\left[N^{2}\left(x_{3}\right) w_{3} \vec{e}_{3}\right], \quad B_{12} y:=P_{0}\left[N^{2}\left(x_{3}\right)\left(\left(U C^{-1 / 2} y\right) \vec{e}_{3}\right) \vec{e}_{3}\right], \\
& B_{21} \vec{w}:=C^{-1 / 2} P_{H_{0}} \Psi, \quad \rho_{0}^{-1} \nabla \Psi=P_{h, S}\left[N^{2}\left(x_{3}\right) w_{3} \vec{e}_{3}\right], \\
& B_{22} y:=C^{-1 / 2} P_{H_{0}} \eta, \quad \rho_{0}^{-1} \nabla \eta=P_{h, S}\left[N^{2}\left(x_{3}\right)\left(\left(U C^{-1 / 2} y\right) \vec{e}_{3}\right) \vec{e}_{3}\right] .
\end{aligned}
$$

In what follows, all functions of the variable $t$ and spatial variables will be regarded as functions of a single variable $t$ with values in the corresponding Hilbert spaces; this was taken into considerations in the above constructions. Because of this below all the derivatives $\partial / \partial t$ are replaced by $d / d t$.

Initial boundary value problem (18) splits into trivial relation (24) and Cauchy problem for a second order differential equation (incomplete, that is, involving no first order terms) in the Hilbert space $\mathcal{H}=\vec{J}_{0}\left(\Omega, \rho_{0}\right) \oplus H_{0}$ :

$$
\begin{array}{ll}
\frac{d^{2}}{d t^{2}} \mathcal{A} \mathcal{X}+(\mathcal{C}+\mathcal{B}) \mathcal{X}=\mathcal{F}, & \mathcal{X}(0)=\mathcal{X}^{0}, \quad \mathcal{X}^{\prime}(0)=\mathcal{X}^{1}, \\
\mathcal{A}=\left(\begin{array}{cc}
I & 0 \\
0 & I+\rho_{2} C^{-1}
\end{array}\right), & \mathcal{C}=\left(\begin{array}{cc}
0 & 0 \\
0 & g \rho_{0} C^{-1}
\end{array}\right), \quad \mathcal{B}=\left(\begin{array}{ll}
B_{11} & B_{12} \\
B_{21} & B_{22}
\end{array}\right), \\
\mathcal{F}=\left(\begin{array}{c}
P_{0} \psi_{0} \\
C^{-1 / 2} P_{H_{0}} F
\end{array}\right), \quad \mathcal{X}=\left(\begin{array}{l}
\vec{w} \\
y
\end{array}\right) . &
\end{array}
$$

Lemma 5. The operator matrix $\mathcal{B}$ in (48) possesses the properties $\mathcal{O} \leqslant \mathcal{B} \leqslant N_{0}^{2} \mathcal{I}$, where $N_{0}^{2}$ is the constant in (1), $\mathcal{O}$ and $\mathcal{I}$ are zero and identity operators in $\mathcal{H}=\vec{J}_{0}\left(\Omega, \rho_{0}\right) \oplus H_{0}$.

Proof. We have

$$
\begin{aligned}
(\mathcal{B X}, \mathcal{X})_{\mathcal{H}} & =\left(\left(\begin{array}{ll}
B_{11} & B_{12} \\
B_{21} & B_{22}
\end{array}\right)\left(\begin{array}{l}
\vec{w} \\
y
\end{array}\right),\left(\begin{array}{l}
\vec{w} \\
y
\end{array}\right)\right)_{\mathcal{H}} \\
& =\left(B_{11} \vec{w}, \vec{w}\right)_{\vec{J}_{0}\left(\Omega, \rho_{0}\right)}+\left(B_{12} y, \vec{w}\right)_{\vec{J}_{0}\left(\Omega, \rho_{0}\right)}+\left(B_{21} \vec{w}, y\right)_{H_{0}}+\left(B_{22} y, y\right)_{H_{0}} .
\end{aligned}
$$

In view of definitions (46), (19), (31), and the Green's formula for the Laplace operator (see, for instance, [15]), we have

$$
\begin{aligned}
& \left(B_{11} \vec{w}, \vec{w}\right)_{\vec{J}_{0}\left(\Omega, \rho_{0}\right)}=\left(P_{0}\left[N^{2}\left(x_{3}\right) w_{3} \vec{e}_{3}\right], \vec{w}\right)_{\vec{J}_{0}\left(\Omega, \rho_{0}\right)}=\int_{\Omega} N^{2}\left(x_{3}\right) \cdot \rho_{0}\left(x_{3}\right)\left|w_{3}\right|^{2} d \Omega \\
& \left(B_{12} y, \vec{w}\right)_{\vec{J}_{0}\left(\Omega, \rho_{0}\right)}=\left(P_{0}\left[N^{2}\left(x_{3}\right)\left(\left(U C^{-1 / 2} y\right) \vec{e}_{3}\right) \vec{e}_{3}\right], \vec{w}\right)_{\vec{J}_{0}\left(\Omega, \rho_{0}\right)}
\end{aligned}
$$




$$
\begin{aligned}
& =\int_{\Omega} N^{2}\left(x_{3}\right) \rho_{0}\left(x_{3}\right)\left(\rho_{0}^{-1} \nabla \Phi \cdot \vec{e}_{3}\right) w_{3} d \Omega \\
\left(B_{21} \vec{w}, y\right)_{H_{0}}= & \left(C^{-1 / 2} P_{H_{0}} \Psi, y\right)_{H_{0}}=\left(P_{H_{0}} \Psi, C^{-1 / 2} y\right)_{H_{0}} \int_{\Gamma} \rho_{0}(0) P_{H_{0}} \Psi\left(\rho_{0}^{-1} \frac{\partial \Phi}{\partial x_{3}}\right)_{\Gamma} d \Gamma \\
= & \int_{\Omega} \rho_{0}\left(x_{3}\right) \cdot \rho_{0}^{-1} \nabla \Psi \cdot \rho_{0}^{-1} \nabla \Phi d \Omega=\int_{\Omega} \rho_{0}\left(x_{3}\right) \cdot N^{2}\left(x_{3}\right) w_{3} \vec{e}_{3} \cdot \rho_{0}^{-1} \nabla \Phi d \Omega \\
= & \int_{\Omega} N^{2}\left(x_{3}\right) \rho_{0}\left(x_{3}\right)\left(\rho_{0}^{-1} \nabla \Phi \cdot \vec{e}_{3}\right) w_{3} d \Omega,{ }_{\Gamma} \rho_{0}(0) P_{H_{0}} \eta\left(\rho_{0}^{-1} \frac{\partial \Phi}{\partial x_{3}}\right)_{\Gamma} d \Gamma \\
\left(B_{22} y, y\right)_{H_{0}}= & \left(C^{-1 / 2} P_{H_{0}} \eta, y\right)_{H_{0}}=\left(P_{H_{0}} \eta, C^{-1 / 2} y\right)_{H_{0}}=\int_{\Omega} \rho_{\Omega_{0}}\left(x_{3}\right) \cdot \rho_{0}^{-1} \nabla \eta \cdot \rho_{0}^{-1} \nabla \Phi d \Omega \\
= & \int_{\Omega} \rho_{0}\left(x_{3}\right) \cdot N^{2}\left(x_{3}\right)\left(\left(U C^{-1 / 2} y\right) \vec{e}_{3}\right) \vec{e}_{3} \cdot \rho_{0}^{-1} \nabla \Phi d \Omega \\
= & \int_{\Omega} N^{2}\left(x_{3}\right) \rho_{0}\left(x_{3}\right)\left|\left(\rho_{0}^{-1} \nabla \Phi \cdot \vec{e}_{3}\right)\right|^{2} d \Omega .
\end{aligned}
$$

On one hand, employing (1) and (21), we get

$$
(\mathcal{B X}, \mathcal{X})_{\mathcal{H}}=\int_{\Omega} N^{2}\left(x_{3}\right) \rho_{0}\left(x_{3}\right)\left|w_{3}+\left(\rho_{0}^{-1} \nabla \Phi \cdot \vec{e}_{3}\right)\right|^{2} d \Omega \geqslant N_{\min }^{2} \int_{\Omega} \rho_{0}\left(x_{3}\right)\left|v_{3}\right|^{2} d \Omega \geqslant 0 .
$$

On the other hand,

$$
\begin{aligned}
(\mathcal{B} \mathcal{X}, \mathcal{X})_{\mathcal{H}} & \leqslant N_{0}^{2} \cdot \int_{\Omega} \rho_{0}\left(x_{3}\right)\left|w_{3}+\left(\rho_{0}^{-1} \nabla \Phi \cdot \vec{e}_{3}\right)\right|^{2} d \Omega \leqslant N_{0}^{2} \cdot \int_{\Omega} \rho_{0}\left(x_{3}\right)|\vec{v}|^{2} d \Omega \\
& =N_{0}^{2}\left(\|\vec{w}\|_{\vec{J}_{0}\left(\Omega, \rho_{0}\right)}^{2}+\left\|\rho_{0}^{-1} \nabla \Phi\right\|_{\vec{G}_{h, S}\left(\Omega, \rho_{0}\right)}^{2}\right) \\
& =N_{0}^{2}\left(\|\vec{w}\|_{\vec{J}_{0}\left(\Omega, \rho_{0}\right)}^{2}+\int_{\Omega} \rho_{0}\left(x_{3}\right) \cdot \rho_{0}^{-1} \nabla \Phi \cdot \rho_{0}^{-1} \nabla \Phi d \Omega\right) \\
& =N_{0}^{2}\left(\|\vec{w}\|_{\vec{J}_{0}\left(\Omega, \rho_{0}\right)}^{2}-\int_{\Omega} \Phi \cdot \operatorname{div}\left(\rho_{0}^{-1} \nabla \Phi\right) d \Omega+\left.\int_{\partial \Omega}(\Phi)\right|_{\Gamma} \cdot\left(\rho_{0}^{-1} \nabla \Phi \cdot \vec{n}\right) d S\right) \\
& =N_{0}^{2}\left(\|\vec{w}\|_{\vec{J}_{0}\left(\Omega, \rho_{0}\right)}^{2}+\left.\int_{\Gamma}(\Phi)\right|_{\Gamma} \cdot\left(\rho_{0}^{-1} \frac{\partial \Phi}{\partial x_{3}}\right)_{\Gamma} d \Gamma\right) \\
& =N_{0}^{2}\left(\|\vec{w}\|_{\vec{J}_{0}\left(\Omega, \rho_{0}\right)}^{2}+\left(C^{1 / 2} y, C^{-1 / 2} y\right)_{H_{0}}\right)=N_{0}^{2}\left(\|\vec{w}\|_{\vec{J}_{0}\left(\Omega, \rho_{0}\right)}^{2}+\|y\|_{H_{0}}^{2}\right)=N_{0}^{2}\|\mathcal{X}\|_{\mathcal{H}}^{2} .
\end{aligned}
$$

Definition 1. A function $\mathcal{X}(t)$ defined on the segment $[0, T]$ is called a strong solution to problem 47$)$ with values in $\mathcal{H}=\vec{J}_{0}\left(\Omega, \rho_{0}\right) \oplus H_{0}$ if the following conditions are satisfied:

$1^{\circ} . \mathcal{X}(t) \in \overrightarrow{J_{0}}\left(\Omega, \rho_{0}\right) \oplus \mathcal{D}\left(C^{-1}\right)$; 
$2^{\circ} . \mathcal{B X}(t) \in C([0, T] ; \mathcal{H}), \quad \mathcal{C X}(t) \in C([0, T] ; \mathcal{H})$

$3^{\circ} . \mathcal{A X}(t) \in C^{2}([0, T] ; \mathcal{H})$;

$4^{\circ}$. the equation and initial conditions in (47) are satisfied.

Theorem 1. Assume that problem (47) satisfies the conditions

$$
\mathcal{X}^{0} \in \vec{J}_{0}\left(\Omega, \rho_{0}\right) \oplus H_{\Gamma}^{1 / 2}, \quad \mathcal{X}^{1} \in \vec{J}_{0}\left(\Omega, \rho_{0}\right) \oplus H_{\Gamma}^{1 / 2}, \quad \mathcal{F} \in C([0, T] ; \mathcal{H}) .
$$

Then this problem has the unique strong solution on the segment $[0, T]$.

Proof. We make the change $C^{-1 / 2} y=z$ in problem (47) and we apply the operator $\operatorname{diag}\left(I ; C^{1 / 2}\right)$ to both sides of equation (47). As a result, we arrive at the following Cauchy problem

$$
\begin{aligned}
& \mathcal{A}_{1} \frac{d^{2}}{d t^{2}} \mathcal{X}_{1}+\mathcal{C}_{B} \mathcal{X}_{1}=\mathcal{F}_{1}, \quad \mathcal{X}_{1}(0)=\mathcal{X}_{1}^{0}, \quad \mathcal{X}_{1}^{\prime}(0)=\mathcal{X}_{1}^{1}, \\
& \mathcal{A}_{1}=\left(\begin{array}{cc}
I & 0 \\
0 & C+\rho_{2} I
\end{array}\right), \quad \mathcal{C}_{B}=\left(\begin{array}{cc}
B_{11} & B_{12} C^{1 / 2} \\
C^{1 / 2} B_{21} & g \rho_{0} I+C^{1 / 2} B_{22} C^{1 / 2}
\end{array}\right), \\
& \mathcal{X}_{1}=\left(\begin{array}{c}
\vec{w} \\
z
\end{array}\right)=\left(\begin{array}{c}
\vec{w} \\
C^{-1 / 2} y
\end{array}\right), \quad \mathcal{F}_{1}=\left(\begin{array}{cc}
I & 0 \\
0 & C^{1 / 2}
\end{array}\right) \mathcal{F} \text {. }
\end{aligned}
$$

The above constructions (Lemmata 4, 5) imply that

$$
0 \ll \mathcal{A}_{1}=\mathcal{A}_{1}^{*} \in \mathcal{L}(\mathcal{H}), \quad 0 \leqslant \mathcal{C}_{B}=\mathcal{C}_{B}^{*} \in \mathcal{L}(\mathcal{H}) .
$$

Then Cauchy problem (51) is strongly solvable if

$$
\mathcal{X}_{1}^{0} \in \mathcal{H}, \quad \mathcal{X}_{1}^{1} \in \mathcal{H}, \quad \mathcal{F}_{1} \in C([0, T] ; \mathcal{H}) .
$$

The proof of this fact was given in [17].

Thus, in view of (53), we obtain conditions (50) of solvability of problem (47). For instance,

$$
\begin{aligned}
\mathcal{X}_{1}^{0}=\left(\begin{array}{c}
\vec{w}^{0} \\
C^{-1 / 2} y^{0}
\end{array}\right) \in \mathcal{H} & =\vec{J}_{0}\left(\Omega, \rho_{0}\right) \oplus H_{0} \\
& \Longleftrightarrow \mathcal{X}^{0}=\left(\begin{array}{c}
\vec{w}^{0} \\
y^{0}
\end{array}\right) \in \vec{J}_{0}\left(\Omega, \rho_{0}\right) \oplus \mathcal{D}\left(C^{-1 / 2}\right)=\vec{J}_{0}\left(\Omega, \rho_{0}\right) \oplus H_{\Gamma}^{1 / 2} .
\end{aligned}
$$

In view of formulations of problems (13), (18), we give (mutually according) definitions of so-called strong in the variable $t$ solutions to these problems.

Definition 2. A strong (in the variable $t$ ) solution to problem (13) on the segment $[0, T]$ is the set of functions $\vec{u}(t, x), p(t, x), \rho(t, x)$ and $\zeta(t, \hat{x})$ obeying the following conditions:

$1^{\circ} . \vec{u}(t) \in C^{1}\left([0, T] ; \vec{J}_{0, S}\left(\Omega, \rho_{0}\right)\right), \rho_{0}^{-1} \nabla p \in C\left([0, T] ; \vec{G}\left(\Omega, \rho_{0}\right)\right), \rho(t) \in C^{1}\left([0, T] ; \mathfrak{L}_{2}(\Omega)\right)$, where $\mathfrak{L}_{2}(\Omega)$ is a Hilbert space of scalar functions with the scalar product

$$
(\varphi, \psi)_{\mathfrak{L}_{2}(\Omega)}:=g^{2} \int_{\Omega}\left[\rho_{0}\left(x_{3}\right) N^{2}\left(x_{3}\right)\right]^{-1} \varphi(x) \overline{\psi(x)} d \Omega
$$

and for each $t \in[0, T]$ the first equation in (13) holds;

$2^{\circ} . u_{n}=\frac{\partial \zeta}{\partial t} \in C\left([0, T] ; H_{0}\right)$;

$3^{\circ}$. the boundary condition on $\Gamma$ holds:

$$
p=g \rho_{0}(0) \zeta+\rho_{2} \frac{\partial^{2} \zeta}{\partial t^{2}} \in C\left([0, T] ; H_{0}\right),
$$

where all terms are continuous in $t$ functions with values in $H_{0}$. 
$4^{\circ}$. initial conditions (13) hold.

Definition 3. A strong (in the variable $t$ ) solution to problem (18) on the segment $[0, T]$ is the set of functions $\vec{v}(t, x), p(t, x)$ obeying the following conditions:

$1^{\circ} . \quad \vec{v}(t) \in C^{2}\left([0, T] ; \vec{J}_{0, S}\left(\Omega, \rho_{0}\right)\right), \quad \rho_{0}^{-1} \nabla p \in C\left([0, T] ; \vec{G}\left(\Omega, \rho_{0}\right)\right)$ and for each $t \in[0, T]$ the first equation in $(18)$ is satisfied;

$2^{\circ}$. boundary condition on $\Gamma$ is satisfied:

$$
p=g \rho_{0}(0) v_{3}+\rho_{2} \frac{\partial^{2} v_{3}}{\partial t^{2}} \in C\left([0, T] ; H_{0}\right), \quad v_{3}=\vec{v} \cdot \vec{e}_{3}
$$

where all terms are continuous in $t$ functions with values in $H_{0}$;

$3^{\circ}$. constraints (15) and (16) hold true;

$4^{\circ}$. initial conditions (18) are satisfied.

Definition 4. A strong (in the variable $t$ ) solution to problem (28) on the segment $[0, T]$ is functions $\vec{w}(t, x)$ in $\vec{J}_{0}\left(\Omega, \rho_{0}\right)$ and $\Phi(t, x)$ with values in $H_{\Gamma}^{1}\left(\Omega, \rho_{0}\right)$, for which the following conditions hold:

$1^{\circ} . \quad \vec{w}(t) \in C^{2}\left([0, T] ; \vec{J}_{0}\left(\Omega, \rho_{0}\right)\right) ;$

$2^{\circ} .\left(\frac{\partial \Phi}{\partial x_{3}}\right)_{\Gamma} \in C^{2}\left([0, T] ; H_{0}\right), \Phi_{\Gamma} \in C^{2}\left([0, T] ; H_{\Gamma}^{1 / 2}\right)$, for all $t \in[0, T] ;$

$3^{\circ}$. the relations

$$
\begin{aligned}
& \frac{\partial^{2} \vec{w}}{\partial t^{2}}+P_{0}\left[N^{2}\left(x_{3}\right)\left(\rho_{0}^{-1} \frac{\partial \Phi}{\partial x_{3}}+w_{3}\right) \vec{e}_{3}\right]=P_{0} \psi_{0} \quad(\text { in } \Omega), \\
& \frac{\partial^{2} \Phi}{\partial t^{2}}+g \frac{\partial \Phi}{\partial x_{3}}+\rho_{2} \rho_{0}^{-1} \frac{\partial^{2}}{\partial t^{2}}\left(\frac{\partial \Phi}{\partial x_{3}}\right)+\Psi+\eta=F+c(t) \quad(\text { on } \Gamma),
\end{aligned}
$$

hold, where all terms are continuous in $t$ functions with values in $\vec{J}_{0}\left(\Omega, \rho_{0}\right)$ and $H_{0}$ respectively and $\rho_{0}^{-1} \nabla \Phi \in C^{2}\left([0, T] ; \vec{G}_{h, S}\left(\Omega, \rho_{0}\right)\right)$;

$4^{\circ}$. the initial conditions

$$
\begin{aligned}
& \frac{\partial}{\partial t}\left(\rho_{0}^{-1} \frac{\partial \Phi}{\partial x_{3}}(0, \hat{x})\right)_{\Gamma}=\left[\left(P_{h, S} \vec{u}^{0}(x)\right) \cdot \vec{n}\right]_{\Gamma} \in H_{0}, \\
& \left(\rho_{0}^{-1} \frac{\partial \Phi}{\partial x_{3}}(0, \hat{x})\right)_{\Gamma}=\zeta(0, \hat{x}) \in H_{0} ; \\
& \frac{\partial}{\partial t}(\vec{w}(0, x))=P_{0} \vec{u}^{0}(x) \in \vec{J}_{0}\left(\Omega, \rho_{0}\right) ; \\
& \vec{w}(0, x)=P_{0} \vec{u}^{0}(x) \in \vec{J}_{0}\left(\Omega, \rho_{0}\right), \quad\left(w_{3}(0, \hat{x})\right)_{\Gamma}=0
\end{aligned}
$$

are satisfied.

Theorem 2. Assume that the conditions

$$
\begin{gathered}
\vec{u}^{0} \in \vec{J}_{0, S}\left(\Omega, \rho_{0}\right), \quad \rho^{0} \in \mathfrak{L}_{2}(\Omega), \quad \zeta^{0} \in H_{0}=L_{2}(\Gamma) \ominus\left\{1_{\Gamma}\right\}, \\
{\left[\left(P_{h, S} \vec{u}^{0}(x)\right) \cdot \vec{n}\right]_{\Gamma} \in H_{0}, \quad f(t) \in C\left([0, T] ; \vec{L}_{2}\left(\Omega, \rho_{0}\right)\right)}
\end{gathered}
$$

hold. Then each of problems (13), (18) and (28) has the unique strong in t solution. 
Proof. We note that choosing the norms $H_{\Gamma}^{1 / 2}$ by (36), (42), by the isometry of the operator $C^{-1}: H_{\Gamma}^{1 / 2}=\mathcal{D}\left(C^{-1}\right) \rightarrow \widetilde{H}_{\Gamma}^{-1 / 2}$ we obviously have the following statements:

$$
\begin{aligned}
\left\{\rho_{0}^{-1} \nabla \Phi\right. & \left.\in C^{k}\left([0, T] ; \vec{G}_{h, S}\left(\Omega, \rho_{0}\right)\right)\right\} \Leftrightarrow\left\{\left.\Phi\right|_{\Gamma} \in C^{k}\left([0, T] ; H_{\Gamma}^{1 / 2}\right)\right\} \\
& \Leftrightarrow\left\{\left.\rho_{0}^{-1} \frac{\partial \Phi}{\partial n}\right|_{\Gamma} \in C^{k}\left([0, T] ; \widetilde{H}_{\Gamma}^{-1 / 2}\right)\right\}, \quad k=0,1,2, \ldots
\end{aligned}
$$

We prove the theorem via several steps passing from problem (47) to (28), then from (28) to (18) and from (18) to (13).

From problem (47) to (28). Assume that conditions (50) holds for the functions $\mathcal{X}^{0}, \mathcal{X}^{1}$ and $\mathcal{F}$ in the theorem on solvability f problem 47$)$. Then

1. The function $\vec{w}(t, x)$ satisfies:

$$
\begin{aligned}
\left(\vec{w}^{0} \in \vec{J}_{0}\left(\Omega, \rho_{0}\right),\right. & \left.\vec{w}^{1} \in \vec{J}_{0}\left(\Omega, \rho_{0}\right)\right) \\
\Leftrightarrow & \left(\left(w_{3}(0, \hat{x})\right)_{\Gamma}=0, \quad \frac{\partial}{\partial t}(\vec{w}(0, x))=P_{0} \vec{u}^{0}(x) \in \vec{J}_{0}\left(\Omega, \rho_{0}\right) .\right)
\end{aligned}
$$

Moreover, $\vec{w}(t) \in C^{2}\left([0, T] ; \vec{J}_{0}\left(\Omega, \rho_{0}\right)\right)$.

2. If problem (47) has the unique strong solution on the segment $[0, T]$ and $y \in H_{\Gamma}^{1 / 2}$, in view of (37) and (44) we have

$$
\left.\Phi\right|_{\Gamma}=C\left(\rho_{0}^{-1} \frac{\partial \Phi}{\partial n}\right)_{\Gamma} \in C^{2}\left([0, T] ; H_{\Gamma}^{1 / 2}\right), \quad\left(\rho_{0}^{-1} \frac{\partial \Phi}{\partial n}\right)_{\Gamma} \in C^{2}\left([0, T] ; H_{0}\right) .
$$

This implies that the function $\Phi=\Phi(t, x)$ satisfy the equation and boundary conditions in problem (28) and all functions in the boundary condition on $\Gamma$ belong to the space $C\left([0, T] ; H_{0}\right)$. It follows from (56) that

$$
\rho_{0}^{-1} \nabla \Phi(t, x) \in C^{2}\left([0, T] ; \vec{G}_{h, S}(\Omega)\right)
$$

Moreover, by (57),

$$
\begin{aligned}
& \left(\rho_{0}^{-1} \frac{\partial \Phi}{\partial x_{3}}(0, x)\right)_{\Gamma}=\zeta^{0}(\hat{x})=C^{-1 / 2} y^{0} \in H_{0}, \\
& C^{-1 / 2} y^{1}=\frac{\partial}{\partial t}\left(\rho_{0}^{-1} \frac{\partial \Phi}{\partial x_{3}}(0, \hat{x})\right)_{\Gamma}=\left[\left(P_{h, S} \vec{u}^{0}(x)\right) \cdot \vec{n}\right]_{\Gamma} \in H_{0},
\end{aligned}
$$

Hence, by Lemma 2 we obtain that

$$
\frac{\partial}{\partial t}\left(\rho_{0}^{-1} \nabla \Phi\right)(0, x)=P_{h, S} \vec{u}^{0} \in \vec{G}_{h, S}\left(\Omega, \rho_{0}\right) .
$$

Therefore, according definition 4 , the functions $\vec{w}(t, x)$ and $\Phi(t, x)$ are a strong solution (with respect to the variable $t$ ) to problem (28) on the segment $[0, T]$.

From problem (28) to (18). Let us make sure that the proven facts imply the existence of a strong (with respect to the variable $t$ ) solution to problem (18). Following an inverse way of transformations (see (21)), by strong solution $\vec{w}(t, x)$ and $\Phi(t, x)$ to problem (28) we introduce the functions $\vec{v}(t, x)$ and $p(t, x)$ :

$$
\vec{v}(t, x)=\vec{w}(t, x)+\rho_{0}^{-1} \nabla \Phi(t, x) \in C^{2}\left([0, T] ; \vec{J}_{0, S}\left(\Omega, \rho_{0}\right)\right),
$$

since

$$
\vec{w}(t, x) \in C^{2}\left([0, T] ; \vec{J}_{0}\left(\Omega, \rho_{0}\right)\right), \quad \rho_{0}^{-1} \nabla \Phi(t, x) \in C^{2}\left([0, T] ; \vec{G}_{h, S}\left(\Omega, \rho_{0}\right)\right)
$$


and

$$
\begin{aligned}
\left.p_{1}\right|_{\Gamma}= & \left.g \rho_{0}(0) v_{3}\right|_{\Gamma}+\left.\rho_{2} \frac{\partial^{2}}{\partial t^{2}} v_{3}\right|_{\Gamma}=g \rho_{0}(0)\left(\rho_{0}^{-1} \frac{\partial \Phi}{\partial x_{3}}\right)_{\Gamma} \\
& +\rho_{2} \frac{\partial^{2}}{\partial t^{2}}\left(\rho_{0}^{-1} \frac{\partial \Phi}{\partial x_{3}}\right)_{\Gamma} \in C\left([0, T] ; H_{0}\right),
\end{aligned}
$$

Therefore, $\rho_{0}^{-1} \nabla p_{1}(x, t) \in C\left([0, T] ; \vec{G}_{h, S}\left(\Omega, \rho_{0}\right)\right)$, and in view (24),

$$
\rho_{0}^{-1} \nabla p(t, x)=\rho_{0}^{-1} \nabla p_{1}(t, x)+\rho_{0}^{-1} \nabla p_{2}(t, x) \in C\left([0, T] ; \vec{G}\left(\Omega, \rho_{0}\right)\right),
$$

Initial condition in problem (28) give rise to initial conditions for problem $(18)$ :

$$
v_{3}(0, \hat{x})=\zeta^{0}(\hat{x}) \in H_{0}, \quad \frac{\partial}{\partial t} \vec{v}(0, x)=\frac{\partial}{\partial t}\left(\vec{w}+\rho_{0}^{-1} \nabla \Phi\right)(0, x)=\vec{u}^{0} \in \vec{J}_{0, S}\left(\Omega, \rho_{0}\right) .
$$

From problem (18) to problem (13). Due to the above proven facts and taking into consideration constraints (15), (16), it is easy to confirm that under the assumptions of the theorem, problem (13) has a strong (with respect to the variable $t$ ) solution in the sense of definition 2 .

In conclusion we observe that

$$
\begin{aligned}
\mathcal{F} & =\left(P_{0} \psi_{0}, C^{-1 / 2} P_{H_{0}} F_{f}\right)^{t} \in C([0, T] ; \mathcal{H}) \\
& \Longleftrightarrow \quad P_{0} \psi_{0} \in C\left([0, T] ; \vec{J}_{0}\left(\Omega, \rho_{0}\right)\right), \quad C^{-1 / 2} P_{H_{0}} F_{f} \in C\left([0, T] ; H_{0}\right) \\
& \Longleftrightarrow \quad P_{0} \psi_{0} \in C\left([0, T] ; \vec{J}_{0}\left(\Omega, \rho_{0}\right)\right), \quad P_{H_{0}} F_{f} \in C\left([0, T] ; H_{\Gamma}^{1 / 2}\right) \\
& \Longleftrightarrow \quad P_{0} \psi_{0} \in C\left([0, T] ; \vec{J}_{0}\left(\Omega, \rho_{0}\right)\right), \quad P_{h, S} \psi_{0}=\rho_{0}^{-1} \nabla F \in C\left([0, T] ; \vec{G}_{h, S}\left(\Omega, \rho_{0}\right)\right) .
\end{aligned}
$$

In view of separated trivial relation (24) we obtain:

$$
\psi_{0} \in C\left([0, T] ; \vec{L}_{2}\left(\Omega, \rho_{0}\right)\right) .
$$

The latter is true if and only if $f(t) \in C\left([0, T] ; \vec{L}_{2}\left(\Omega, \rho_{0}\right)\right)$, see 17$)$.

The authors thank the referee for attention to the work and remarks.

\section{BIBLIOGRAPHY}

1. W. Krauss. Internal waves. Gidrometeoizdat, Leningrad (1968). [Interne Wellen, Gebruder Bornträger, Berlin (1966). (in German).]

2. J.S. Turner. Buoyancy effects in fluids. Cambridge Univ. Press, Cambridge (1973)

3. V.M. Kamenkovich. Fundamentals of ocean dynamics. Gidrometeoizdat, Leningrad (1973). [Elsevier, Amsterdam (1977).]

4. Yu.Z. Miropol'sky. Dynamics of internal gravity waves in the ocean. Gidrometeoizdat, Leningrad (1981). [Springer, Dordrecht (2001).]

5. S.A. Gabov, A.G. Sveshnikov. Problems in the dynamics of stratified fluids. Nauka, Moscow (1986). (in Russian).

6. S.A. Gabov, A.G. Sveshnikov. Linear problems of the theory of non-stationary internal waves. Nauka, Moscow (1990). (in Russian).

7. N.D. Kopachevskii, A.N. Temnov. Vibrations of a stratified liquid in a basin of arbitrary shape // Zurn. Vychisl. Matem. Matem. Fiz. 26:5, 734-755 (1986). [USSR Comp. Math. Math. Phys. 26:3, 58-72 (1986).]

8. N.D. Kopachevskii, D.O. Tsvetkov. Oscillations of stratificated fluids // J. Math. Sci 164:4, 574$602(2010)$. 
9. S.A. Gabov. A hydrodynamics problem related to flotation for a perfect fluid // Differ. Uravn. 24:1, 16-21 (1988). [Diff. Equat. 24:1, 12-15 (1988).]

10. S.A. Gabov, A.G. Sveshnikov. Mathematical problems of the dynamics of a floating fluid // Itogi Nauki Tekhn. Ser. Matem. Anal. 28, 3-86 (1990). [J. Soviet Math. 54:4, 979-1041 (1991).]

11. M.A. Soldatov. Mathematical aspects of theory of liquid oscillations in a basin partially covered by ice. PhD thesis, Kharkiv (2003). (in Russian).

12. L.D. Landau, E.M. Lifschitz. Hydrodynamics. Nauka, Moscow (1986). [Fluid Mechanics. Pergamon Press, Oxford (1987).]

13. L.M. Brekhovskii, V.V. Goncharov. Introduction in mechanics of continuous media. Nauka, Moscow (1982). (in Russian).

14. N.D. Kopachevskii. Abstract Green's formula and some of its applications. "Forma", Ltd., Simferopol (2016). (in Russia).

15. N.D. Kopachevskii, S.G. Krein, Zuj Kan Ngo. Operator methods in hydrodynamics. Evolutionary and spectral problems. Nauka, Moscow (1989).

16. E. Gagliardo. Caratterizzazioni delle tracce sulla frontiera relative ad alcune classi di funzioni in $n$ variabili // Rend. Semin. Matem. Univ. Padova. 27, 284-305 (1957).

17. N.D. Kopachevskii. Integro-differential Volterra equations in Hilbert space: Special lecture course. "Bondarenko O.A.", Simferopol (2012). (in Russian).

Nikolai Dmitrievich Kopachevskii,

V.I. Vernadsky Crimean Federal University, acad. Vernadsky av. 4, 295000, Simferopol, Russia

E-mail: kopachevsky@list.ru

Denis Olegovich Tsvetkov,

V.I. Vernadsky Crimean Federal University, acad. Vernadsky av. 4,

295000, Simferopol, Russia

E-mail: tsvetdo@gmail.com 